\title{
Application of the Multistep Generalized Differential Transform Method to Solve a Time-Fractional Enzyme Kinetics
}

\author{
Ahmed Alawneh \\ Department of Science and Humanities, Princess Sumaya University for Technology, Amman 11941, Jordan \\ Correspondence should be addressed to Ahmed Alawneh; aalawneh@ju.edu.jo
}

Received 29 September 2012; Accepted 21 December 2012

Academic Editor: R. Sahadevan

Copyright (C) 2013 Ahmed Alawneh. This is an open access article distributed under the Creative Commons Attribution License, which permits unrestricted use, distribution, and reproduction in any medium, provided the original work is properly cited.

\begin{abstract}
The multistep differential transform method is first employed to solve a time-fractional enzyme kinetics. This enzyme-substrate reaction is formed by a system of nonlinear ordinary differential equations of fractional order. The fractional derivatives are described in the Caputo sense. A comparative study between the new algorithm and the classical Runge-Kutta method is presented in the case of integer-order derivatives. The results demonstrate reliability and efficiency of the algorithm developed.
\end{abstract}

\section{Introduction}

Mathematical modeling of complex processes is a major challenge for contemporary scientists. In contrast to simple classical systems, where the theory of integer-order differential equations is sufficient to describe their dynamics, complex systems are characterized by the variability of structures in them, multiscale behavior, and nonlinearity in the mathematical description of the mutual relationship between parameters [1]. Fractional derivatives provide an excellent instrument for the description of dynamical behavior of various complex materials and systems. There are two important benefits: (1) we have more degrees of freedom in the model considered and (2) a "memory" is enclosed in the model. Therefore, the number of scientific and engineering problems involving fractional derivatives is already very large and still growing and perhaps the fractional calculus (i.e., derivatives and integrals of any real or complex order) will be the calculus of the twenty-first century [1].

Recent investigations have shown that many complex biological systems can be represented more accurately through fractional derivative formulation. Magin [2] was the first who used fractional derivatives and fractional integrals in order to model stress-strain relationship in biomaterials. Craiem et al. [3] applied fractional calculus to model arterial viscoelasticity. Abdullah [4] used fractional differential equations to model the Michaelis-Menten reaction in a $2 \mathrm{~d}$ region containing obstacles. He investigated the phenomenon of subdiffusion, which occurs when there is molecular crowding, by proposing a continuous spatial model involving fractional differential equations. The Michaelis-Menten mechanism is the simplest chemical network which models the formation of a product through an enzymatic catalysis of a substrate. In particular, an enzyme reacts with the substrate and reversibly forms an intermediate complex, which then decays into the product and original enzyme. This enzyme reaction model is given by (for more details on the enzyme dynamics, see [5-9] and the references therein)

$$
E+S \underset{k_{-1}}{\stackrel{k_{1}}{\longrightarrow}} E S \stackrel{k_{2}}{\longrightarrow} E+P
$$

where $E$ is the enzyme, $S$ the substrate, $E S$ the enzymesubstrate intermediate complex, and $P$ the product. The parameters $k_{1}, k_{-1}$, and $k_{2}$ are positive rate constants and denote the rates of reaction of these three processes. Note that substrate binding is reversible but product release is not. The concentration of the reactants in (1) is denoted by lower case letters:

$$
s=[S], \quad e=[E], \quad c=[S E], \quad p=[P] .
$$

Applying the law of mass action, which states that reaction rates are proportional to the concentrations of the reactants, 
the time evolution of scheme (1) can be determined from the solution of the system of coupled nonlinear ordinary differential equations (ODEs) [6, 7]:

$$
\begin{gathered}
\frac{d s}{d t}=-k_{1} e s+k_{-1} c, \\
\frac{d e}{d t}=-k_{1} e s+\left(k_{-1}+k_{2}\right) c, \\
\frac{d c}{d t}=k_{1} e s-\left(k_{-1}+k_{2}\right) c, \\
\frac{d p}{d t}=k_{2} c,
\end{gathered}
$$

subject to the initial conditions

$$
s(0)=s_{0}, \quad e(0)=e_{0}, \quad c(0)=0, \quad p(0)=0 .
$$

Systems (3) can be reduced to only two equations for $s$ and $c$, namely [6],

$$
\begin{gathered}
\frac{d s}{d t}=-k_{1} e_{0} s+\left(k_{1} s+k_{-1}\right) c \\
\frac{d c}{d t}=k_{1} e_{0} s-\left(k_{1} s+k_{-1}+k_{2}\right) c
\end{gathered}
$$

subject to the initial conditions $s(0)=s_{0}$ and $c(0)=0$. By introducing the following parameters:

$$
\begin{gathered}
\tau=\frac{k_{1} e_{0} t}{\varepsilon}, \quad u(\tau)=\frac{s(t)}{s_{0}}, \quad v(\tau)=\frac{c(t)}{c_{0}}, \\
w(\tau)=\frac{p(t)}{e_{0}}, \quad \lambda=\frac{k_{2}}{k_{1} s_{0}}, \quad k_{3}=\frac{k_{-1}+k_{2}}{k_{1} s_{0}}, \quad \varepsilon=\frac{e_{0}}{s_{0}} .
\end{gathered}
$$

Then, (5) and the initial conditions (4) can be represented in dimensionless form as follows:

$$
\begin{gathered}
\frac{d u}{d t}=-\varepsilon u+\varepsilon\left(u+k_{3}-\lambda\right) v, \\
\frac{d v}{d t}=u-\left(u+k_{3}\right) v, \\
\frac{d w}{d t}=\lambda v, \\
u(0)=1, \quad v(0)=0, \quad w(0)=0,
\end{gathered}
$$

where $\varepsilon, k$, and $\lambda$ are dimensionless parameters.

Now, we introduce fractional order into enzyme reaction model. The new system is described by the following set of fractional differential equations:

$$
\begin{gathered}
D^{\alpha_{1}} u=-\varepsilon u+\varepsilon\left(u+k_{3}-\lambda\right) v, \\
D^{\alpha_{2}} v=u-\left(u+k_{3}\right) v, \\
D^{\alpha_{3}} w=\lambda v, \\
u(0)=1, \quad v(0)=0, \quad w(0)=0,
\end{gathered}
$$

where the fractional derivatives $D^{\alpha_{1}} u, D^{\alpha_{2}} v$, and $D^{\alpha_{3}} w$ are considered in the Caputo sense, and $0<\alpha_{i} \leq 1, i=$ $1,2,3$, are parameters describing the order of the fractional time-derivatives in the Caputo sense. The general response expression contains parameters describing the order of the fractional derivatives that can be varied to obtain various responses. Obviously, the classical integer-order enzyme reaction model can be viewed as a special case from the fractional-order enzyme reaction model by setting $\alpha_{1}=\alpha_{2}=$ $\alpha_{3}=1$. In other words, the ultimate behavior of the fractional system response must converge to the response of the integerorder version of the equation [10].

Our motivation for this work is to obtain the approximate solution of the time-fractional enzyme kinetics (8)-(10) using the multistep generalized differential transform method (MSGDTM). This method is only a simple modification of the generalized differential transform method (GDTM) [11-14], in which it is treated as an algorithm in a sequence of small intervals (i.e., time step) for finding accurate approximate solutions to the corresponding systems. The approximate solutions obtained by using GDTM are valid only for a short time, while the ones obtained by using the MSGDTM $[15,16]$ are more valid and accurate during a long time and are in good agreement with the RK4-5 numerical solution when the order of the derivative $\alpha_{i}=1, i=1,2,3$.

This paper is organized as follows. In Section 2, we present some necessary definitions and notations related to fractional calculus and differential transform method. In Section 3, the proposed method is described. In Section 4, the method is applied to the problems (8)-(10), and numerical simulations are presented graphically. Finally, the conclusions are given in Section 5.

\section{Preliminaries}

In this section, we give some basic definitions and properties of the fractional calculus theory and differential transform method which are used further in this paper $[1,10,17-25]$.

\subsection{Fractional Calculus}

Definition 1. A function $f(x)(x>0)$ is said to be in the space $C_{\alpha}(\alpha \in R)$ if it can be written as $f(x)=x^{p} f_{1}(x)$ for some $p>\alpha$, where $f_{1}(x)$ is continuous in $[0, \infty)$, and it is said to be in the space $C_{\alpha}^{m}$ if $f^{(m)} \in C_{\alpha}, m \in \mathbf{N}$.

Definition 2. The Riemann-Liouville integral operator of order $\alpha>0$ with $a \geq 0$ is defined as

$$
\begin{gathered}
\left(J_{a}^{\alpha} f\right)(x)=\frac{1}{\Gamma(\alpha)} \int_{a}^{x}(x-t)^{\alpha-1} f(t) d t, \quad x>a, \\
\left(J_{a}^{0} f\right)(x)=f(x) .
\end{gathered}
$$


Properties of the operator can be found in $[1,10]$. We only need here the following: for $f \in C_{\alpha}, \alpha, \beta>0, a \geq 0, c \in R$ and $\gamma>-1$, we have

$$
\begin{gathered}
\left(J_{a}^{\alpha} J_{a}^{\beta} f\right)(x)=\left(J_{a}^{\beta} J_{a}^{\alpha} f\right)(x)=\left(J_{a}^{\alpha+\beta} f\right)(x), \\
J_{a}^{\alpha} x^{\gamma}=\frac{x^{\gamma+\alpha}}{\Gamma(\alpha)} B_{(x-a) / x}(\alpha, \gamma+1),
\end{gathered}
$$

where $B_{\tau}(\alpha, \gamma+1)$ is the incomplete beta function which is defined as

$$
\begin{gathered}
B_{\tau}(\alpha, \gamma+1)=\int_{0}^{\tau} t^{\alpha-1}(1-t)^{\gamma} d t, \\
J_{a}^{\alpha} e^{c x}=e^{a c}(x-a)^{\alpha} \sum_{k=0}^{\infty} \frac{[c(x-a)]^{k}}{\Gamma(\alpha+k+1)} .
\end{gathered}
$$

The Riemann-Liouville derivative has certain disadvantages when trying to model real-world phenomena with fractional differential equations. Therefore, we will introduce a modified fractional differential operator $D_{a}^{\alpha}$ proposed by Caputo in his work on the theory of viscoelasticity.

Definition 3. The Caputo fractional derivative of $f(x)$ of order $\alpha>0$ with $a \geq 0$ is defined as

$$
\left(D_{a}^{\alpha} f\right)(x)=\left(J_{a}^{m-\alpha} f^{(m)}\right)(x)=\frac{1}{\Gamma(m-\alpha)} \int_{a}^{x} \frac{f^{(m)}(t)}{(x-t)^{\alpha+1-m}} d t
$$

for $m-1<\alpha \leq m, m \in \mathbf{N}, x \geq a, f(x) \in C_{-1}^{m}$.

The Caputo fractional derivative was investigated by many authors; for $m-1<\alpha \leq m, f(x) \in C_{\alpha}^{m}$ and $\alpha \geq-1$, we have

$$
\left(J_{a}^{\alpha} D_{a}^{\alpha} f\right)(x)=J^{m} D^{m} f(x)=f(x)-\sum_{k=0}^{m-1} f^{(k)}(a) \frac{(x-a)^{k}}{k !} .
$$

For mathematical properties of fractional derivatives and integrals, one can consult the mentioned references.

\subsection{The Definitions and Operations of Differential Transform.} The differential transform technique is one of the seminumerical analytical methods for ordinary and partial differential equations that uses the form of polynomials as approximations of the exact solutions that are sufficiently differentiable. The basic definition and the fundamental theorems of the differential transform method (DTM) and its applicability for various kinds of differential equations are given in [2123]. For convenience of the reader, we present a review of the DTM. The differential transform of the $k$ th derivative of function $f(t)$ is defined as follows:

$$
F(k)=\frac{1}{k !}\left[\frac{d^{k} f(t)}{d t^{k}}\right]_{t=t_{0}}
$$

where $f(t)$ is the original function and $F(k)$ is the transformed function. The differential inverse transform of $F(k)$ is defined as:

$$
f(t)=\sum_{k=0}^{\infty} F(k)\left(t-t_{0}\right)^{k}
$$

From (17) and (18), we get

$$
f(z)=\left.\sum_{k=0}^{\infty} \frac{\left(z-z_{0}\right)^{k}}{k !} \frac{d^{k} f(z)}{d z^{k}}\right|_{z=z_{0}}
$$

which implies that the concept of differential transform is derived from Taylor series expansion, but the method does not evaluate the derivatives symbolically. However, relative derivatives are calculated by an iterative way which is described by the transformed equations of the original function. For implementation purposes, the function $f(t)$ is expressed by a finite series, and (18) can be written as

$$
f(t) \approx \sum_{k=0}^{N} F(k)\left(t-t_{0}\right)^{k}
$$

Here, $N$ is decided by the convergence of natural frequency. The fundamental operations performed by differential transform can readily be obtained and are listed in Table 1 . The main steps of the DTM, as a tool for solving different classes of nonlinear problems, are the following. First, we apply the differential transform (17) to the given problem (integral equation, ordinary differential equation, or partial differential equations), and then the result is a recurrence relation. Second, solving this relation and using the differential inverse transform (18), we can obtain the solution of the problem.

\section{Solving the Systems (8)-(10) Using the Multistep Generalized Differential Transform Method (MSGDTM)}

It has been shown that the approximated solutions obtained using DTM are not valid for large $t$ for some systems [1114]. Therefore, we use the multistep generalized differential transform method (MSGDTM) to solve the system of equations (8)-(10). The method is only a simple modification of the generalized differential transform method, in which it is treated as an algorithm in a sequence of small intervals (i.e., time step) for finding accurate approximate solutions to the corresponding systems $[15,16]$. This MSGDTM offers accurate solutions over a longer time frame (more stable) compared to the standard GDTM. 
TABLE 1: Operations of differential transformation.

\begin{tabular}{|c|c|}
\hline Original function & Transformed function \\
\hline$f(t)=u(t) \pm v(t)$ & $F(k)=U(k) \pm V(k)$ \\
\hline$f(t)=\alpha u(t)$ & $F(k)=\underset{k}{\alpha U(k)}$ \\
\hline$f(t)=u(t) v(t)$ & $F(k)=\sum_{l=0}^{\kappa} U(l) V(k-l)$ \\
\hline$f(t)=\frac{d u(t)}{d t}$ & $F(k)=(k+1) U(k+1)$ \\
\hline$f(t)=\frac{d^{m} u(t)}{d t^{m}}$ & $F(k)=(k+1)(k+2) \cdots(k+m) U(k+m)$ \\
\hline$f(t)=\int_{t_{0}}^{t} u(t) d t$ & $F(k)=\frac{U(k-1)}{k}, k \geq 1$ \\
\hline$f(t)=t^{m}$ & $F(k)=\delta(k-m)= \begin{cases}1, & k=m \\
0, & k \neq m\end{cases}$ \\
\hline$f(t)=\exp (\lambda t)$ & $F(k)=\frac{\lambda^{k}}{k !}$ \\
\hline$f(t)=\sin (\omega t+\alpha)$ & $F(k)=\frac{\omega^{k}}{k !} \sin \left(\frac{\pi k}{2}+\alpha\right)$ \\
\hline$f(t)=\cos (\omega t+\alpha)$ & $F(k)=\frac{\omega^{k}}{k !} \cos \left(\frac{\pi k}{2}+\alpha\right)$ \\
\hline$h(t)=\frac{f(t)}{g(t)}$ & $H(k)=\frac{1}{G(0)}\left[F(k)-\sum_{m=0}^{k-1} H(m) G(k-m)\right]$ \\
\hline$f(t)=[g(t)]^{b}$ & $F(k)= \begin{cases}G(0), & k=0 \\
\sum_{m=1}^{k} \frac{(b+1) m-k}{k G(0)} G(m) F(k-m), & k \geq 1\end{cases}$ \\
\hline
\end{tabular}

Taking the differential transform of (8)-(10) with respect to time $t$ gives

$$
\begin{aligned}
& U(k+1) \\
& =\frac{\Gamma(\alpha k+1)}{\Gamma(\alpha(k+1)+1)} \\
& \quad \times\left[-\varepsilon U(k)+\varepsilon\left(\sum_{l=0}^{k} U(l) V(k-l)+\left(k_{3}-\lambda\right) V(k)\right)\right], \\
& V(k+1) \\
& =\frac{\Gamma(\alpha k+1)}{\Gamma(\alpha(k+1)+1)} \\
& \quad \times\left[U(k)-\sum_{l=0}^{k} U(l) V(k-l)-k_{3} V(k)\right] \\
& \quad W(k+1)=\frac{\Gamma(\alpha k+1)}{\Gamma(\alpha(k+1)+1)} \lambda V(k),
\end{aligned}
$$

where $U(k), V(k)$, and $W(k)$ are the differential transformation forms of $u(t), v(t)$, and $w(t)$, respectively. The differential transform forms of the initial conditions are given by $U(0)=$ $1, V(0)=0$, and $W(0)=0$. In view of the differential inverse transform, the differential transform series solution for the systems (8)-(10) can be obtained as

$$
\begin{aligned}
& u(t)=\sum_{n=0}^{N} U(n) t^{\alpha n}, \\
& v(t)=\sum_{n=0}^{N} V(n) t^{\alpha n}, \\
& w(t)=\sum_{n=0}^{N} W(n) t^{\alpha n} .
\end{aligned}
$$

Now, according to the multistep generalized differential transform method, the series solution for the systems (8)-(10) is suggested by

$$
u(t)= \begin{cases}\sum_{n=0}^{K} U_{1}(n) t^{\alpha n}, & t \in\left[0, t_{1}\right], \\ \sum_{n=0}^{K} U_{2}(n)\left(t-t_{1}\right)^{\alpha n}, & t \in\left[t_{1}, t_{2}\right], \\ \vdots & \\ \sum_{n=0}^{K} U_{M}(n)\left(t-t_{M-1}\right)^{\alpha n}, & t \in\left[t_{M-1}, t_{M}\right],\end{cases}
$$




$$
\begin{gathered}
v(t)= \begin{cases}\sum_{n=0}^{K} V_{1}(n) t^{\alpha n}, & t \in\left[0, t_{1}\right], \\
\sum_{n=0}^{K} V_{2}(n)\left(t-t_{1}\right)^{\alpha n}, & t \in\left[t_{1}, t_{2}\right], \\
\vdots & \\
\sum_{n=0}^{K} V_{M}(n)\left(t-t_{M-1}\right)^{\alpha n}, & t \in\left[t_{M-1}, t_{M}\right],\end{cases} \\
w(t)= \begin{cases}\sum_{n=0}^{K} W_{1}(n) t^{\alpha n}, & t \in\left[0, t_{1}\right], \\
\sum_{n=0}^{K} W_{2}(n)\left(t-t_{1}\right)^{\alpha n}, & t \in\left[t_{1}, t_{2}\right], \\
\vdots & \\
\sum_{n=0}^{K} W_{M}(n)\left(t-t_{M-1}\right)^{\alpha n}, & t \in\left[t_{M-1}, t_{M}\right],\end{cases}
\end{gathered}
$$

where $U_{i}(n), V_{i}(n)$, and $W_{i}(n)$ for $i=1,2, \ldots, M$ satisfy the following recurrence relations:

$$
\begin{aligned}
& U_{i}(k+1) \\
& =\frac{\Gamma(\alpha k+1)}{\Gamma(\alpha(k+1)+1)} \\
& \times\left[-\varepsilon U_{i}(k)+\varepsilon\left(\sum_{l=0}^{k} U_{i}(l) V_{i}(k-l)+\left(k_{3}-\lambda\right) V_{i}(k)\right)\right], \\
& V_{i}(k+1) \\
& =\frac{\Gamma(\alpha k+1)}{\Gamma(\alpha(k+1)+1)} \\
& \quad \times\left[U_{i}(k)-\sum_{l=0}^{k} U_{i}(l) V_{i}(k-l)-k_{3} V_{i}(k)\right], \\
& \quad W_{i}(k+1)=\frac{\Gamma(\alpha k+1)}{\Gamma(\alpha(k+1)+1)} \lambda V_{i}(k),
\end{aligned}
$$

such that $U_{i}(0)=U_{i-1}(0), V_{i}(0)=V_{i-1}(0)$, and $W_{i}(0)=$ $W_{i-1}(0)$. Finally, if we start with $U_{0}(0)=1, V_{0}(0)=0$, and $W_{0}(0)=0$, using the recurrence relation given in (24), then we can obtain the multistep solution given in (23).

This multistage MSGDTM offers accurate solutions over a longer time frame (more stable) compared to the standard GDTM [11-14]. This distinctive strategy grants the iterative algorithm a time-marching scheme which significantly drives forward the convergence of the solutions precisely with great rapidity. The value of $t$ increases according to the designated time-step size in each iteration computation. Each amplified time step will produce a new approximation value for the desired iteration step, and the process is continued until the targeted time frame is achieved. Errors are therefore

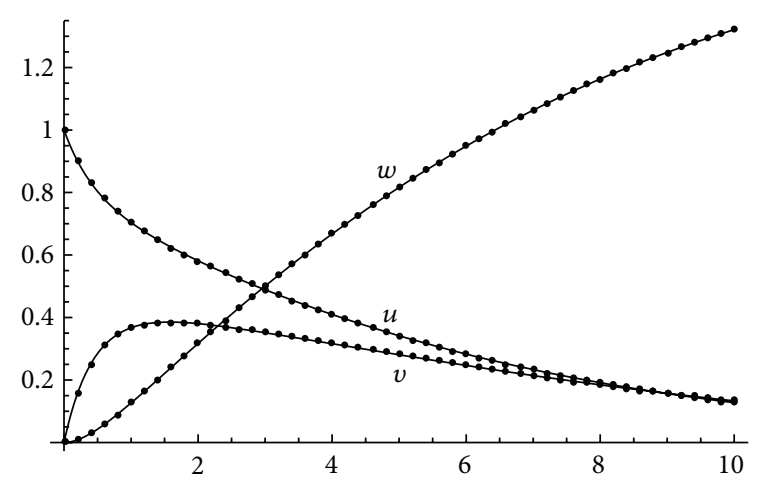

FIGURE 1: Profile of the normalized concentrations of the substrate $u$, enzyme-substrate complex $v$, and product $w$ for $k=1, \lambda=0.5$, $\varepsilon=0.6$, and $\alpha_{1}=\alpha_{2}=\alpha_{3}=1$. MSDTM solution (solid line); RungeKutta method solution (dotted line).

minimized and depend on the time step $\Delta t$, but the main disadvantage lies in having longer computational time. Generally, the convergence is achieved more quickly as the iteration steps increase.

\section{Numerical Results}

In this work, we carefully propose the MSGDTM, a reliable modification of the GDTM, that improves the convergence of the series solution. The method provides immediate and visible symbolic terms of analytic solutions, as well as numerical approximate solutions to both linear and nonlinear differential equations. To demonstrate the effectiveness of the proposed algorithm as an approximate tool for solving the nonlinear system of fractional differential equations (8)-(10) for larger $t$, we apply the proposed algorithm on the interval $[0,10]$. We divide this interval to subintervals with time step $\Delta t=0.1$ or $M=100$ and the tem-number of GDTM series solutions is fixed, $K=10$. All the results are calculated by using the computer algebra package Mathematica.

Figures 1 and 2 show the approximate solutions obtained using the MSGDTM and the fourth-order Runge-Kutta method of concentrations of substrate $u$, enzyme-substrate complex $v$, and product $w$ for various values of dimensionless reaction parameters $k_{3}, \lambda$, and $\varepsilon$ when $\alpha_{1}=\alpha_{2}=\alpha_{3}=1$. It can be seen that the results from the MSGDTM match the results of the Runge-Kutta method very well, which implies that the MSGDTM can predict the behavior of these variables accurately for the region under consideration. Therefore, the proposed method is a very efficient and accurate method that can be used to provide analytical solutions for nonlinear systems of differential equations.

Next, we consider how the concentrations of substrate $u$, enzyme-substrate complex $v$, and product $w$ depend upon the magnitude of the order of fractional derivatives. We fix the dimensionless reaction parameters $k_{3}, \lambda$, and $\varepsilon$ and perform the numerical simulation for different values of $\alpha_{1}, \alpha_{2}$, and $\alpha_{3}$. Simulation results are presented in Figures 3, 4, 5, and 6, and 


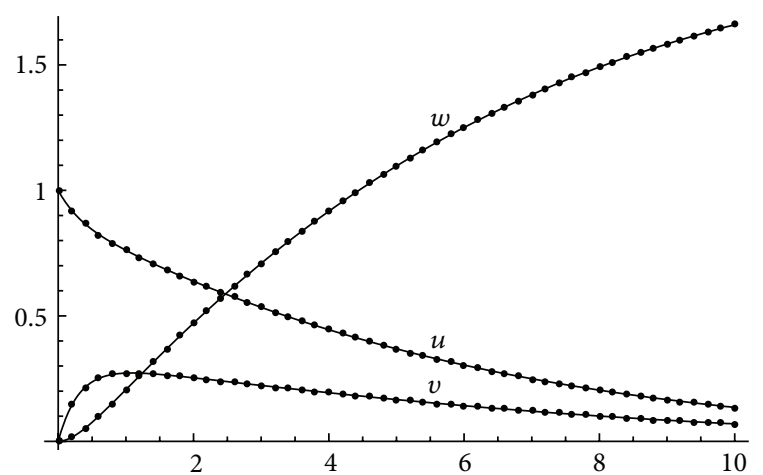

FIgURE 2: Profile of the normalized concentrations of the substrate $u$, enzyme-substrate complex $v$, and product $w$ for $k_{3}=2, \lambda=1.0$, $\varepsilon=0.5$, and $\alpha_{1}=\alpha_{2}=\alpha_{3}=1$. MSDTM solution (solid line); RungeKutta method solution (dotted line).

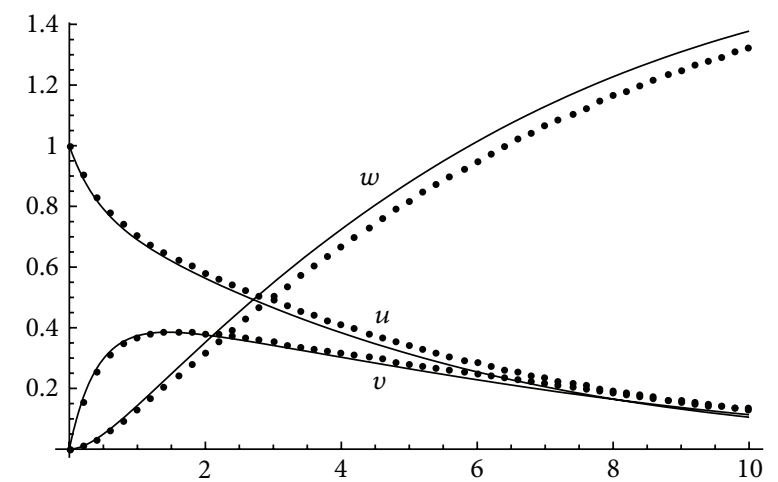

FIGURE 3: Profile of the normalized concentrations of the substrate $u$, enzyme-substrate complex $v$, and product $w$ for $k_{3}=1, \lambda=0.5$, and $\varepsilon=0.6, \alpha_{1}=0.98, \alpha_{2}=0.98$, and $\alpha_{3}=0.98$ (solid line); $\alpha_{1}=$ $\alpha_{2}=\alpha_{3}=1$ (dotted line).

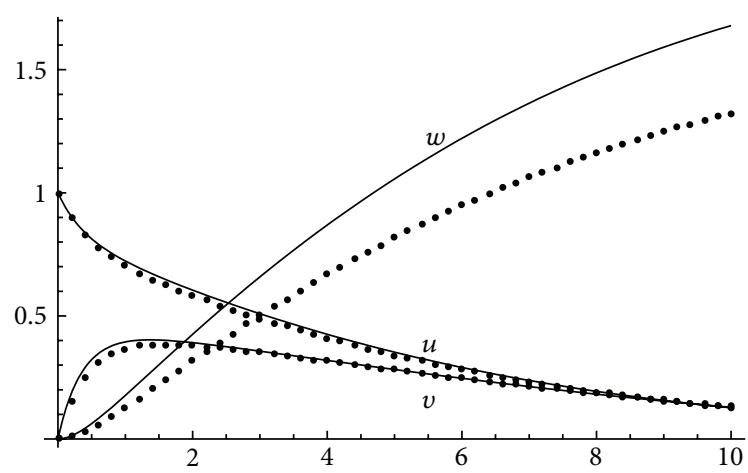

FIgURE 4: Profile of the normalized concentrations of the substrate $u$, enzyme-substrate complex $v$, and product $w$ for $k_{3}=1, \lambda=0.5$, and $\varepsilon=0.6, \alpha_{1}=1.0, \alpha_{2}=0.95$, and $\alpha_{3}=0.95$ (solid line); $\alpha_{1}=$ $\alpha_{2}=\alpha_{3}=1$ (dotted line).

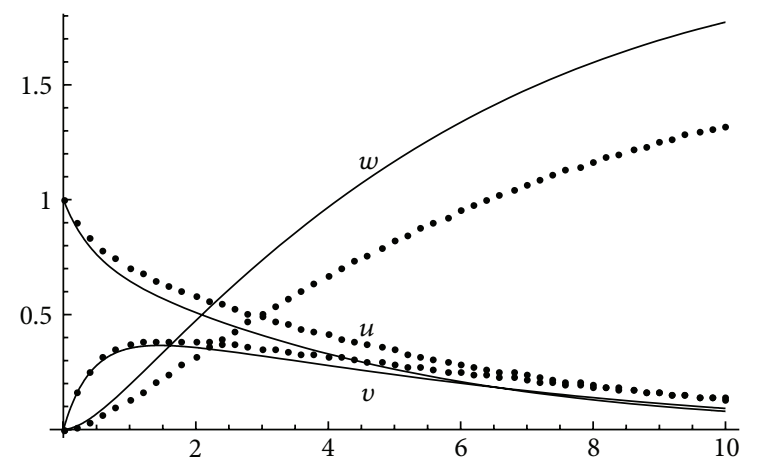

Figure 5: Profile of the normalized concentrations of the substrate $u$, enzyme-substrate complex $v$, and product $w$ for $k_{3}=1, \lambda=0.5$, and $\varepsilon=0.6, \alpha_{1}=0.95, \alpha_{2}=1.0$, and $\alpha_{3}=0.9$ (solid line); $\alpha_{1}=\alpha_{2}=$ $\alpha_{3}=1$ (dotted line).

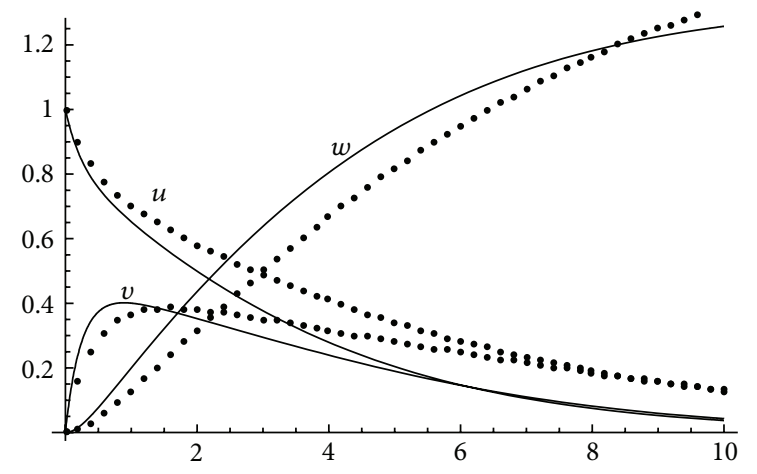

FIGURE 6: Profile of the normalized concentrations of the substrate $u$, enzyme-substrate complex $v$, and product $w$ for $k_{3}=1, \lambda=0.5$, and $\varepsilon=0.6, \alpha_{1}=0.9, \alpha_{2}=0.85$, and $\alpha_{3}=0.95$ (solid line); $\alpha_{1}=$ $\alpha_{2}=\alpha_{3}=1$ (dotted line).

it is clear that the concentrations of the substrate $u$, enzymesubstrate complex $v$, and product $w$ continuously depend on the fractional derivatives. For example, the concentration of substrate $u$ decreases gradually from its initial value of the concentration $(u(0)=1)$ and converges to zero when $\alpha_{1}=\alpha_{2}=\alpha_{3}=1$ and decreases slowly to zero when $\alpha_{1}=0.9, \alpha_{2}=0.85$, and $\alpha_{3}=0.95$. However, the product $w$ increases rapidly for the integer-order derivatives and increases slowly in the case of fractional-order derivatives as shown in Figures 3-6. The key finding of these graphs is that the generalized enzyme kinetics model with fractional derivatives has more degrees of freedom and therefore can be varied to obtain various responses of the concentrations of substrate $u$, enzyme-substrate complex $v$, and product $w$.

\section{Conclusions}

In this paper, the application of MSGDTM was extended to obtain explicit and numerical solutions of a time-fractional 
enzyme kinetics. The MSGDTM was clearly a very efficient and powerful technique in finding the solutions of the proposed equations. The obtained results demonstrate the reliability of the algorithm and its wider applicability to fractional nonlinear evolution equations.

\section{Acknowledgment}

The author gratefully acknowledges the financial support received from The University of Jordan during the sabbatical leave from Department of Mathematics, The University of Jordan, Amman, Jordan.

\section{References}

[1] J. S. Leszczynski, An Introduction to Fractional Mechanics, Czestochowa University of Technology, 2011.

[2] R. L. Magin, Fractional Calculus in Bioengineering, Begell House, Redding, Calif, USA, 2006.

[3] D. O. Craiem, F. J. Rojo, J. M. Atienza, G. V. Guinea, and R. L. Armentano, "Fractional calculus applied to model arterial Viscoelasticity," Latin American Applied Research, vol. 38, no. 2, pp. 141-145, 2008.

[4] F. A. Abdullah, "Using fractional differential equations to model the Michaelis-Menten reaction in a 2 -d region containing obstacles," ScienceAsia, vol. 37, no. 1, pp. 75-78, 2011.

[5] M. A. Savageau, "Michaelis-Menten mechanism reconsidered: implications of fractal kinetics," Journal of Theoretical Biology, vol. 176, no. 1, pp. 115-124, 1995.

[6] S. Schnell and C. Mendoza, "Closed form solution for timedependent enzyme kinetics," Journal of Theoretical Biology, vol. 187, no. 2, pp. 207-212, 1997.

[7] A. Meena, A. Eswari, and L. Rajendran, "Mathematical modelling of enzyme kinetics reaction mechanisms and analytical solutions of non-linear reaction equations," Journal of Mathematical Chemistry, vol. 48, no. 2, pp. 179-186, 2010.

[8] S. M. Goh, M. S. M. Noorani, and I. Hashim, "Introducing variational iteration method to a biochemical reaction model," Nonlinear Analysis: Real World Applications, vol. 11, no. 4, pp. 2264-2272, 2010.

[9] I. Hashim, M. S. H. Chowdhury, and S. Mawa, "On multistage homotopy-perturbation method applied to nonlinear biochemical reaction model," Chaos, Solitons and Fractals, vol. 36, no. 4, pp. 823-827, 2008.

[10] I. Podlubny, Fractional Differential Equations, Academic Press, New York, NY, USA, 1999.

[11] Z. Odibat, S. Momani, and V. S. Erturk, "Generalized differential transform method: application to differential equations of fractional order," Applied Mathematics and Computation, vol. 197, no. 2, pp. 467-477, 2008.

[12] S. Momani and Z. Odibat, "A novel method for nonlinear fractional partial differential equations: combination of DTM and generalized Taylor's formula," Journal of Computational and Applied Mathematics, vol. 220, no. 1-2, pp. 85-95, 2008.

[13] Z. Odibat and S. Momani, "A generalized differential transform method for linear partial differential equations of fractional order," Applied Mathematics Letters, vol. 21, no. 2, pp. 194-199, 2008.

[14] V. S. Erturk, S. Momani, and Z. Odibat, "Application of generalized differential transform method to multi-order fractional differential equations," Communications in Nonlinear Science and Numerical Simulation, vol. 13, no. 8, pp. 1642-1654, 2008.

[15] Z. M. Odibat, C. Bertelle, M. A. Aziz-Alaoui, and G. H. E. Duchamp, "A multi-step differential transform method and application to non-chaotic or chaotic systems," Computers and Mathematics with Applications, vol. 59, no. 4, pp. 1462-1472, 2010.

[16] V. S. Ertrk, Z. M. Odibat, and S. Momani, "An approximate solution of a fractional order differential equation model of human T-cell lymphotropic virus i (HTLV-I) infection of $\mathrm{CD}^{4+}$ T-cells," Computers and Mathematics with Applications, vol. 62, no. 3, pp. 996-1002, 2011.

[17] M. Caputo, "Linear models of dissipation whose Q is almost frequency independent-II," Geophysical Journal of the Royal Astronomical Society, vol. 13, no. 5, pp. 529-539, 1967.

[18] A. A. Kilbas, H. M. Srivastava, and J. J. Trujillo, Theory and Applications of Fractional Differential Equations, Elsevier, Amsterdam, The Netherlands, 2006.

[19] R. Hilfer, Applications of Fractional Calculus in Physics, World Scientific, New Jersey, NJ, USA.

[20] R. L. Bagley and P. J. Torvik, "A theoretical basis for the application of fractional calculus," Journal of Rheology, vol. 27, no. 3, pp. 201-210, 1983.

[21] O. Özkan, "Numerical implementation of differential transformations method for integro-differential equations," International Journal of Computer Mathematics, vol. 27, no. 3, 10 pages.

[22] C. W. Bert and H. Zeng, "Analysis of axial vibration of compound bars by differential transformation method," Journal of Sound and Vibration, vol. 275, no. 3-5, pp. 641-647, 2004.

[23] B. Jang, "Solving linear and nonlinear initial value problems by the projected differential transform method," Computer Physics Communications, vol. 181, no. 5, pp. 848-854, 2010.

[24] E. Heinsalu, M. Patriarca, I. Goychuk, G. Schmid, and P. Hänggi, "Fractional fokker-planck dynamics: numerical algorithm and simulations," Physical Review E, vol. 73, no. 4, Article ID 046133, 9 pages, 2006.

[25] E. Heinsalu, M. Patriarca, I. Goychuk, and P. H. Hanggi, "Use and abuse of a fractional fokker-planck dynamics for timedependent driving," Physical Review Letters, vol. 99, no. 12, Article ID 120602, 4 pages, 2007. 


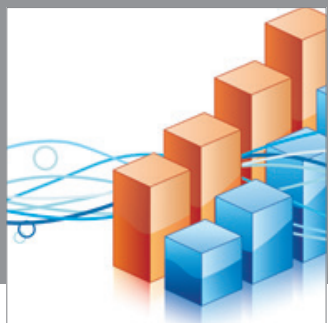

Advances in

Operations Research

mansans

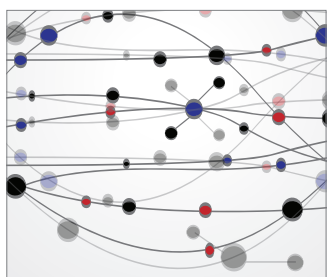

The Scientific World Journal
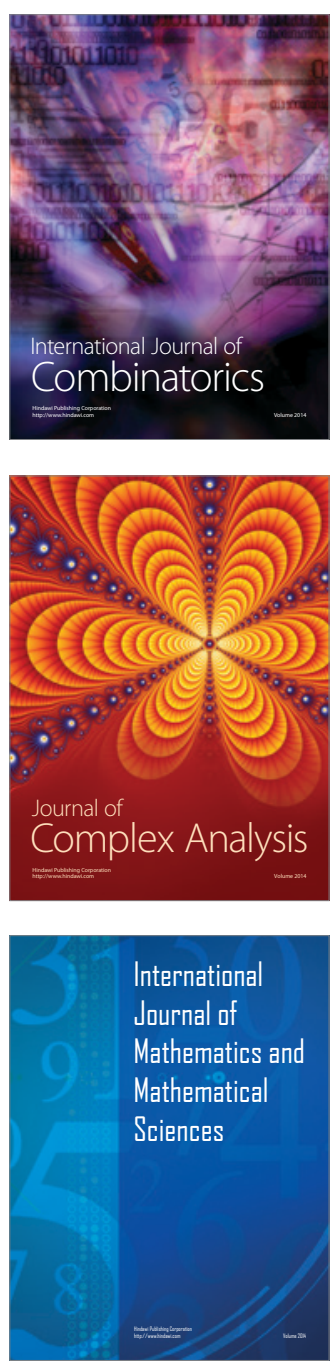
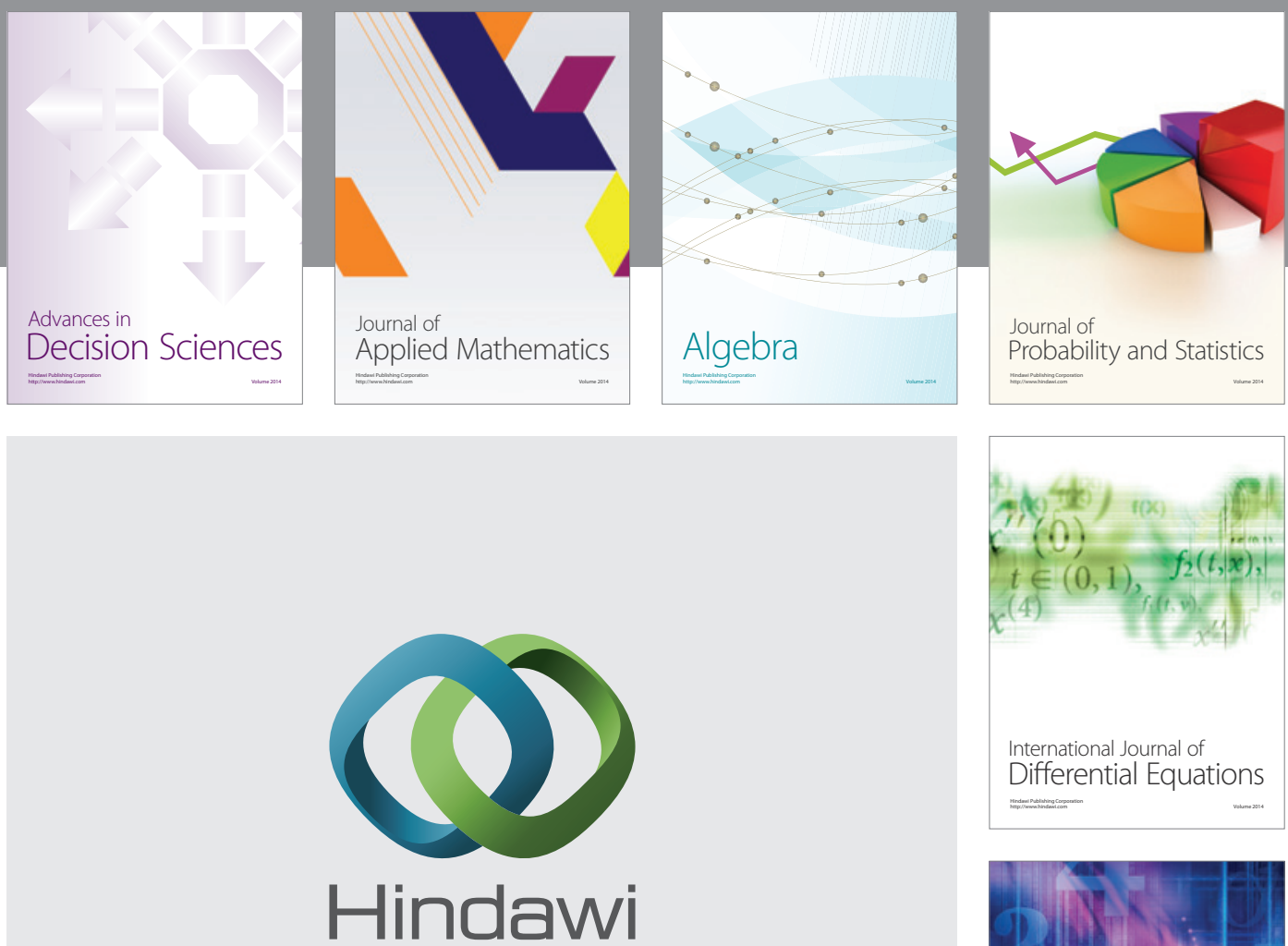

Submit your manuscripts at http://www.hindawi.com
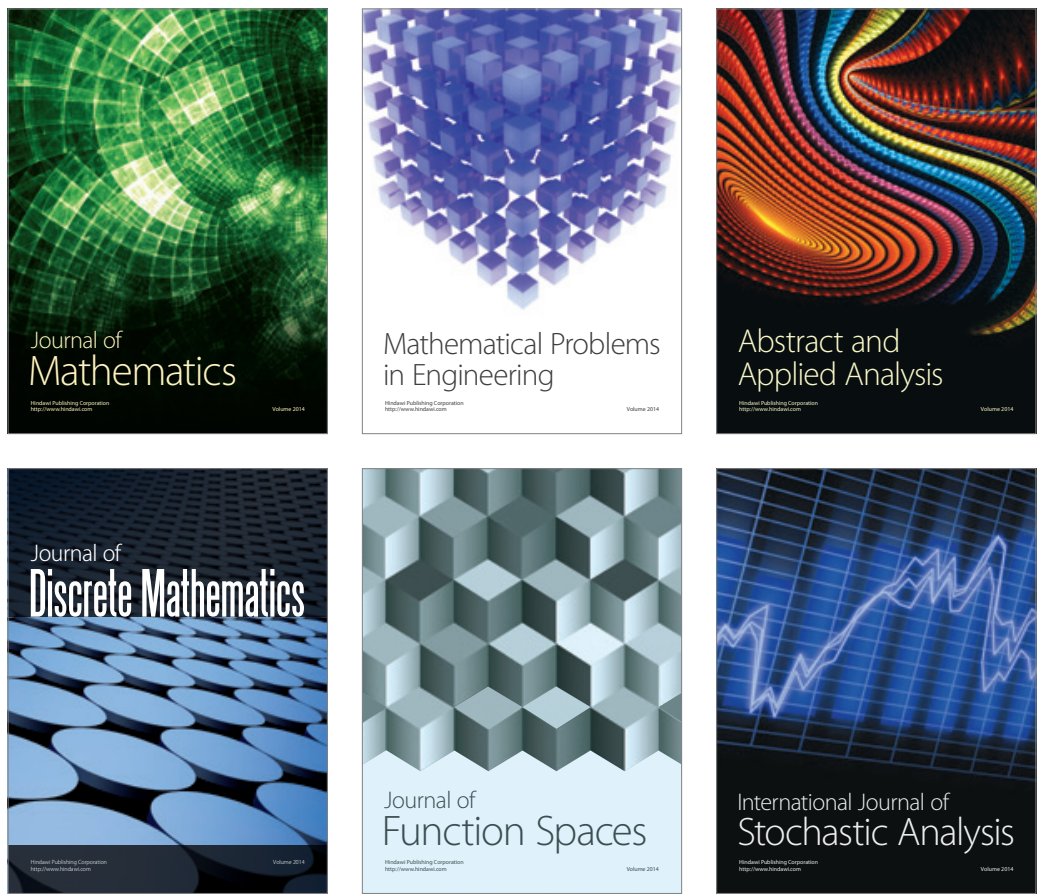

Journal of

Function Spaces

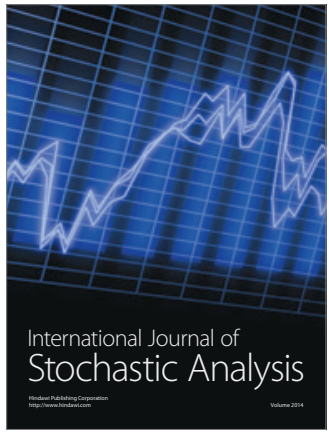

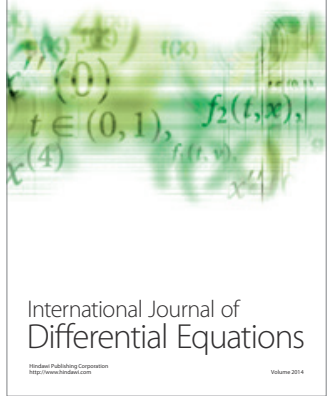
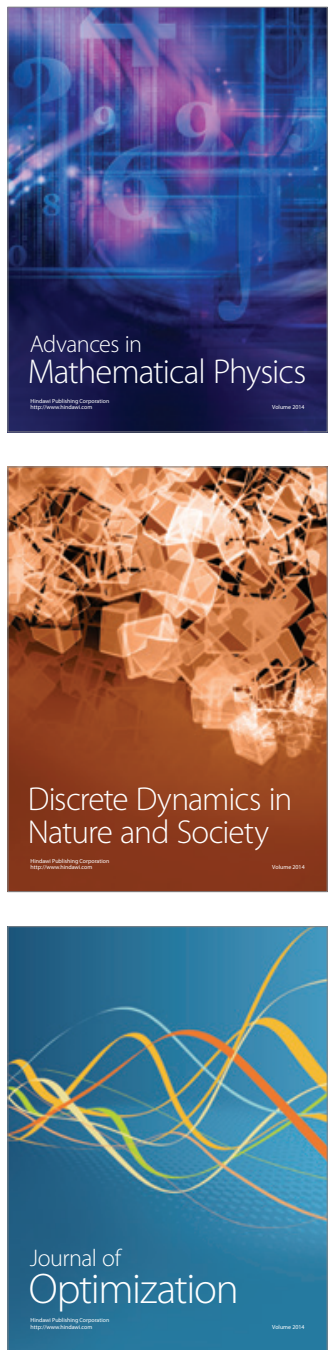